\title{
Preconditioning CS exposure: Variation in place of conditioning and of presentation'
}

D. CHRIS ANDERSON, TIMOTHY O'FARRELL, RICHARD FORMICA, and VICKI CAPONIGRI, University of Notre Dame, Notre Dame, Ind. 46556

Two groups of rats were given preconditioning $C S$ (PCS) exposures in an operant box after leaming to bar press for food. One-half of two other groups were given similar PCS exposures in a holding cage, but otherwise were treated identically. One group that got PCS in the operant box and one that did not were given $10 \mathrm{CS}$-US (buzzer-shock) pairings in the operant chamber while the other two were identically conditioned in a cylindrical chamber. A fifth group got only the US. CS presentations following conditioning produced the most suppression of bar pressing for Ss that got no PCS, intermediate suppression if the PCS was given in the holding cage, and no suppression if preexposures occurred in the operant chamber. These effects were independent of place of conditioning.

Preconditioning CS (PCS) exposures have produced conflicting effects upon acquisition and extinction of a classically conditioned response. For example, Grant, Hake, Riopelle, \& Kostlan (1951) found little statistical support for its effect on classical eyelid learning while, more recently, Lubow \& Moore (1959) and Lubow (1965) reported a decrement in leg flexion conditioning following PCS exposures. Moreover, Anderson, Merrill, Dexter, \& Alleman (1968) and Carlton \& Vogel (1967) reported that the operant performance of fear-conditioned Ss who did not get PCS treatment was more suppressed to the CS during testing than that of similarly conditioned rats who were given PCS exposure.

In an attempt to extend the CER findings of Anderson et al (1968) and Carlton \& Vogel (1967), Anderson, Wolf, \& Sullivan (in press) noted the possibility that place of PCS exposure might be important in demonstrating its degrading effect on performance following conditioning. Although their data in this regard did not quite achieve statistical reliability, their tentative observations raise questions regarding the effect of place of PCS exposure in relation to both the place of conditioning and of testing. The present study evaluated these issues.

\section{SUBJECTS}

The Ss were 30 male naive albino rats, 90-120 days old at the beginning of the experiment. A $6 \frac{3 / 4}{4} \times 2 \frac{3 / 4}{4}$ in. cylindrical chamber composed of molded stainless-stee rings was used for conditioning, and was located inside a darkened, sound-deadened, and ventilated enclosure. The scrambled shock US was $120 \mathrm{~V}$ ac through a $20 \mathrm{~K}$-ohm resistor. The CS was a $6-\mathrm{V}$ ac buzzer $(25 \mathrm{~dB}$ above a $66-\mathrm{dB}$ ambient background level).

Two identical standard operant conditioning boxes containing single levers and recessed food trays were employed for training and testing. These boxes were housed in separate sound-deadened and ventilated observation enclosures. During PCS exposures and fear conditioning the operant boxes were modified slightly by removal of the lever and food tray (openings covered with aluminum inserts). The buzzer CS produced the same sound level output as during conditioning. The Masonite holding cages in which all Ss were housed both before and immediately following sessions in the operant box were $7 \% \times 6 \times 5$ in. inside.

All 30 Ss were trained to a stable lever-press response rate for continuous food reinforcement under $80 \%$ body weight (maintained throughout the study) over seven daily 15-min sessions. Five groups of six Ss were formed, equated for bar-press rate for the last two sessions.

Two groups received three $30-\sec$ PCS exposures over 15-min periods on each of the next 5 days in the operant boxes. While the remaining three groups did not receive PCS exposures in the operant boxes, half of two of these groups were given identical PCS exposures in the holding cages in the 15 -min period prior to or following similar placements in the operant chamber. The averaged physical intensity of the CS was identical for both the holding cages and the operant boxes. The other half of these two and the third groups were not exposed to the CS before conditioning.

Twenty-four hours following the last PCS exposure, four of the five groups were given 10 forward classical aversive conditioning (FC) trials. For one group who received PCS exposures in the operant box and for one who did not, conditioning was conducted in the cylindrical chamber. For the other two similarly-treated groups conditioning was conducted in the operant box. The CS duration was $5 \mathrm{sec}$ and overlapped and coterminated with the 2 -sec US. The intertrial interval averaged $60 \mathrm{sec}( \pm 20 \mathrm{sec})$.

The fifth group was treated identically

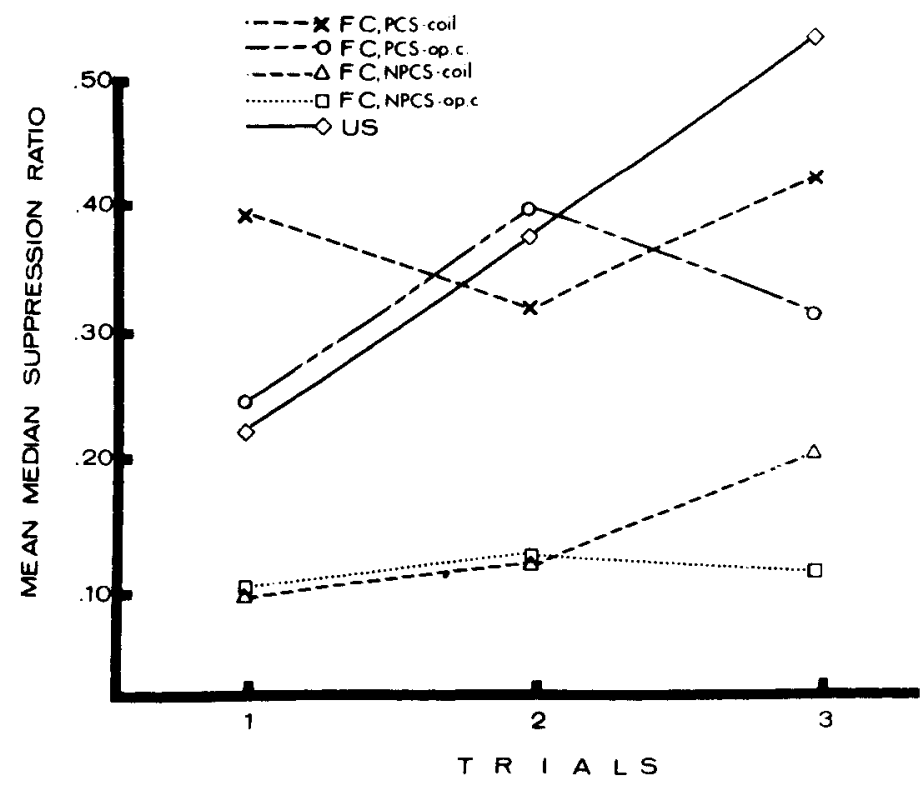

Fig. 1. Mean median suppression ratios for groups who were forward conditioned (FC) in the operant box (op.c.) or coil after either getting or not getting preconditioning CS (PCS or NPCS) exposures in the operant box. 
during conditioning with the exception that the CS was omitted. Hall of the se Sis received their 10 shocks in the cylindrical chamber and the other half in the operant box.

Suppression testing involved return of all Ss to the operant box for continuous food reinforcement, and three $30-\mathrm{sec}$ CS presentations at randomly selected intervals.

\section{RESULTS}

Suppression ratios (SR) were formed by dividing the number of responses during CS presentations (B) by the sum of the responses during the $30-\mathrm{sec}$ period prior to the CS onset $(A)$ and those during $B$ [i.e., $\mathrm{SR}=\mathrm{B} /(\mathbf{A}+\mathbf{B})]$

Since a nested-factors design was employed, several analyses were required to fully evaluated the data. Figure 1 summarizes part of the test results. As seen, the US control group exhibited suppression to the CS on the first, but fully recovered by the third test trial. There was no difference between the performances of this and the two FC (forward conditioned) groups who had received PCS exposures in the operant chamber $(1<1)$. However, the two FC groups who did not get PCS exposures in the operant box exhibited consistently greater test suppression than the other three.

Analyzing the data of all four FC groups, the only significant $F$ was for PCS conditions $(F=14.65$, df $=1 / 20, p<.01)$ thus supporting the observation of relatively greater suppression for the two forward conditioned groups who did not receive PCS exposures in the operant box. Separate comparisons indicated that the PCS (in the operant box) and US control groups differed from the non-PCS, forward conditioned groups (all ps $<.025$ ). (It should be noted that SRs for the respective halves of the US controls were undistinguishable.)

Since the presence or absence of PCS exposures in the holding cages was a nested factor, a separate analysis of these data was conducted. Again, the only reliable effect for this analysis resulted from the greater suppression for the groups who did not receive PCS exposures (overall mean median $\mathrm{SR}=.07$ ) as opposed to those who did (overall mean median $\mathrm{SR}=.19$ ) in the holding cages $(\mathrm{F}=7.30, \mathrm{df}=1 / 10$, $\mathrm{p}<.025$ ).

A comparison of the intermediately suppressed performance of the FC group who received PCS exposures in the holding cages (combined from the Ss conditioned in the box and in the coil) with those of the FC groups who had been given PCS exposures in the operant box indicated reliably less test suppression for the latter ( $F=5.88$, $\mathrm{df}=1 / 15, \mathrm{p}<.05)$.

\section{DISCUSSION}

Place of conditioning does not seem important relative to the location of PCS exposures. Even though the coil and the operant box were highly physically differ- ent, the effect of conditioning in either was identical whe ther PCS exposures occurred in the operant environment or in the holding cage. However, place of PCS exposure appears important. Groups who received preexposures in the test situation exhibited no suppression to test presentations of the CS while comparably conditioned groups who received PCS exposures (identical physical intensity) elsewhere exhibited pronounced suppression. However, PCS exposures still offset conditioning when they occurred outside the test environment. These latter Ss exhibited less suppression that those who did not receive the PCS treatment anywhere.

At least three somewhat different theoretical notions have been suggested to account for the effect of PCS exposures. Carlton \& Vogel (1967) have proffered a habituation notion, but did not offer an account for how a habituated stimulus fails to become conditioned. This interpretation also may encounter a logical difficulty. Thompson \& Spencer (1966) have indicated that a habituated response invariably can be dishabituated by a strong stimulus. Although Thompson \& Spencer (1966) have argued cogently that dishabituation may be a separate process from habituation, their development nevertheless would suggest abrupt dishabituation to the strong shocks used for conditioning.

A second hypothesis is that some sort of contextual discrimination between place of PCS exposure and conditioning may occur, the result being that $S$ may learn that the CS is not a signal for shock in one environment but is in another (cf. Anderson et al, 1968). This interpretation is not confirmed in the present study since the two groups who received PCS exposures in the operant box performed identically even though one was conditioned in the same environment.

Interestingly, Lubow (1965) has proposed that PCS exposures may result in acquisition of responses which are incompatible with classical defensive training. It is difficult to see how this notion can account for the differential effectiveness of PCS exposures in different environments while, at the same time, handle the relative insensitivity of the PCS effect to highly different conditioning situations.

\section{REFERENCES}

ANDERSON, D. C., MERRILL, H. K., DEXTER, W. \& ALLEMAN, H. Contextual effects in emotional learning. Proceedings of the American Psychological Association, 1968, 3, 147-148.

ANDERSON, D. C., WOLF, D., \& SULLIVAN, $P$. Preconditioning exposures to the CS: Variations in place of testing. Psychonomic Science, in press.

CARLTON, P. L., \& VOGEL, J. R. Habituation and conditioning. Journal of Comparative \& Physiological Psy chology, 1967, 63, 348-351.

GRANT, D. A., HAKE, H.W, RIOPELLE, A. J. \& DOSTLAN, A. Effects of repeated pretesting with conditioned stimulus upon extinction of the conditioned eyelid response to light. American Joumal of Psychology, 1951, 54, 247-252.

LUBOW, R. E. Latent inhibition: Effects of frequency of nonreinforced preexposure of the CS. Journal of Comparative \& Physiological Psychology, 1965, 60, 454-457.

LUBOW, R. E., \& MOORE, A. U. Latent inhibition: The effect of nonreinforced preexposure to the conditional stimulus. Journal of Comparative \& Phy siological Psychology, 1959, 52,415-419.

THOMPSON, R. F., \& SPENCER, W. A. Habituation: A model phenomenon for the study of neuronal substrates of behavior. Psy chological Review, 1966, 73, 16-43. NOTE

1. This research was supported by Grant $\mathrm{MH}$ 15364-01 from the National Institute of Mental Health, USPHS.

\section{Behavior of infant rhesus monkeys with thalidomide-induced malformations: A pilot study}

D. G. LINDBURG ${ }^{2}$ National Center for Primate Biology, University of Califormia, Davis, Calif. 95616

Infant monkeys having gross upper limb malformations compensate for manually performed tasks by using the mouth and feet. In overall levels of maternal, peer, and environmental interaction they showed a slight deficit only in exploratory activity in comparison with normal infants.

In concert with a study of thalidomide. induced teratology in thesus monkeys, two infants with gross upper limb deformities were made available for behavioral studies. These animals were bipedal in their locomotion, and could neither cling to the mother without support nor engage in the manipulative activities characteristic of normal monkeys. In addition to a study of locomotor and manipulative skills, a pilot study was designed to permit assessment of maternal-infant behavior and the development of social interaction with peers. There have been no previous reports on the possible effects of thalidomide on the behavior of any nonhuman primate. 